\title{
Éleveurs et agriculteurs du nord du Cameroun face à la
} violence et aux insécurités

Entre adaptation et impuissance

\section{Herders and Farmers of North Cameroon in the Grip of \\ Violence and Insecurity}

Caught Between Adjustment and Powerlessness

Ganaderos y agricultores del norte de Camerún frente a la violencia y a la inseguridad Entre adaptación e impotencia

\author{
Natali Kossoumna Liba’a, Patrick Dugué et Emmanuel Torquebiau
}

Volume 55, numéro 155, septembre 2011

Version originale soumise en mai 2010. Version révisée reçue en avril 2011.

URI : https://id.erudit.org/iderudit/1007225ar

DOI : https://doi.org/10.7202/1007225ar

Aller au sommaire du numéro

Éditeur(s)

Département de géographie de l’Université Laval

ISSN

0007-9766 (imprimé)

1708-8968 (numérique)

Découvrir la revue

\section{Citer cet article}

Liba’a, N. K., Dugué, P. \& Torquebiau, E. (2011). Éleveurs et agriculteurs du nord du Cameroun face à la violence et aux insécurités : entre adaptation et impuissance. Cahiers de géographie du Québec, 55(155), 175-195.

https://doi.org/10.7202/1007225ar
Résumé de l'article

Les insécurités et la violence qui affectent les populations rurales de la province du Nord (Cameroun) trouvent leur origine dans l'histoire et la crise économique qui touche ce pays depuis plus de 20 ans, ainsi que dans les crises politico-militaires fréquentes dans les pays voisins (Tchad, République centrafricaine). Les systèmes de production agropastoraux sont aujourd'hui fragilisés par l'insécurité foncière et fiscale et, plus récemment par des faits de banditisme armé. Les éleveurs Mbororo sédentarisés sont les plus touchés. La difficulté à résoudre les différentes formes d'insécurité provient, d'une part, de l'affaiblissement de l'administration qui ne reçoit pas assez de moyens de l'État et, d'autre part, du manque d'organisation de la société civile et des organismes de producteurs ruraux pour y faire face. 


\title{
Éleveurs et agriculteurs du nord du Cameroun face à la violence et aux insécurités Entre adaptation et impuissance
}

\author{
Herders and Farmers of North Cameroon in \\ the Grip of Violence and Insecurity \\ Caught Between Adjustment and Powerlessness \\ Ganaderos y agricultores del norte de \\ Camerún frente a la violencia y a la \\ inseguridad \\ Entre adaptación e impotencia
}

\author{
Natali KOSSOUMNA LIBA'A \\ Université de Maroua \\ kolibaa@yahoo.fr \\ Patrick DUGUÉ \\ CIRAD, UMR Innovation, Montpellier \\ Patrick.Dugue@cirad.fr \\ Emmanuel TORQUEBIAU \\ CIRAD, UR BSEF et Université de Pretoria \\ Emmanuel.Torquebiau@cirad.fr
}

\section{Résumé}

Les insécurités et la violence qui affectent les populations rurales de la province du Nord (Cameroun) trouvent leur origine dans l'histoire et la crise économique qui touche ce pays depuis plus de 20 ans, ainsi que dans les crises politico-militaires fréquentes dans les pays voisins (Tchad, République centrafricaine). Les systèmes de production agropastoraux sont aujourd'hui fragilisés par l'insécurité foncière et fiscale et, plus récemment par des faits de banditisme armé. Les éleveurs Mbororo sédentarisés sont les plus touchés. La difficulté à résoudre les différentes formes d'insécurité provient, d'une part, de l'affaiblissement de l'administration qui ne reçoit pas assez de moyens de l'État et, d'autre part, du manque d'organisation de la société civile et des organismes de producteurs ruraux pour y faire face.

\section{Mots-clés}

Insécurité, éleveur Mbororo, agriculteur, régime foncier, développement régional, nord du Cameroun.

\begin{abstract}
Today, violence and insecurity continue to plague rural communities in North Cameroon, a situation rooted in its history, in a domestic economic crisis that goes back 20 years, and in the political and military conflicts gripping neighbouring countries like Chad and the Central African Republic. The agro-pastoral systems of production are currently being undermined by land and fiscal insecurity and, more recently, by acts of armed banditry. The sedentary Mbororo herders have been the main victims so far. The difficulty in overcoming the different forms of insecurity stems from the weakness of an administration suffering from government underfunding, and the lack of organization of civil society and rural producer associations preventing North Cameroon from facing up to this situation.
\end{abstract}

\section{Keywords}

Insecurity, Mbororo livestock breeder, farmer, land tenure, regional development, North Cameroon. 


\section{Resumen}

La inseguridad y la violencia que afectan las poblaciones rurales de la provincia del norte de Camerún tienen su origen en la historia y en la crisis económica que aflige el país desde hace más de 20 años, así como en las frecuentes crisis político-militares de los países limítrofes (Tchad, República Centro-africana). Hoy en día, los sistemas de producción agropecuaria son fragilizados por la inseguridad territorial y fiscal y, recientemente, por actos de bandolerismo armado, siendo los sedentarizados ganaderos Mbororo los más afectados. Dos factores explican la dificultad de resolver las diferentes formas de inseguridad: el debilitamiento de la administración que no recibe la ayuda necesaria del Estado; la falta de organización de la sociedad civil y de las organizaciones rurales para confrontarlas.

\section{Palabras Claves}

Inseguridad, ganadero Mbororo, agricultor, desarrollo regional, Norte del Camerún.

\section{Introduction}

Depuis trois décennies, de nombreuses crises sociopolitiques émaillées de faits de violence ont affecté les populations civiles d'Afrique centrale (République centrafricaine, Tchad, République démocratique du Congo, Nigéria et Cameroun). Migrations, insécurités alimentaires et sanitaires, affrontements interethniques, décapitalisation des ménages et des entreprises sont les principales conséquences de ces crises (Bangoura, 2006). D’autres facteurs viennent accroître ce sentiment d'insécurité que ressentent les habitants de ces régions, en particulier les populations rurales : le manque d'emploi, les aléas climatiques (sécheresses, inondations), la variabilité des prix des intrants et des produits agricoles, de même que la dégradation des ressources naturelles. Ainsi, dans les campagnes du nord du Cameroun, les éleveurs peuls Mbororo constituent une population particulièrement vulnérable ${ }^{1}$. Ils subissent un ensemble de facteurs de fragilisation qui ont pour origine l'histoire du peuplement de cette région et la dégradation continue des conditions de production, en particulier la raréfaction des ressources fourragères. À cela s'ajoutent depuis plus d'une décennie, les exactions des "coupeurs de route» qui prennent en otage les enfants et les femmes des éleveurs contre le paiement d'une rançon de plusieurs millions de FCFA (francs de la Communauté financière africaine, 1000 francs CFA = 1,5 euro ou 2,1 dollars US). Les différentes formes d'insécurité touchant les éleveurs Mbororo suscitent des questions sur l'évolution de leur mode de vie, mais plus globalement sur les relations que ces éleveurs entretiennent avec les autres acteurs.

Cet article analyse les causes et conséquences des faits de violence et des diverses formes d'insécurité en milieu rural dans le nord du Cameroun. Il aborde de façon systémique la question de la vulnérabilité des populations rurales en décrivant la diversité des formes de violence et d’insécurité. La violence se rapporte à des faits entre un

1 Dans le texte, le terme Mbororo est synonyme d'«éleveur peul Mbororo». Les Mbororo font partie de la grande famille des Peuls d'Afrique subsaharienne. En milieu rural, ils vivent tous de l'élevage. On ne prendra pas en compte dans ce texte la diversité des clans qui constituent ce groupe social. 
agresseur et un agressé qui portent atteinte à l'intégrité physique ou psychique de ce dernier. L'insécurité désigne le sentiment fait d'anxiété ou de peur que peut ressentir un individu ou une collectivité dans la vie quotidienne devant ce qui peut advenir. Dans ce contexte, comment les éleveurs et les agriculteurs peuvent-ils s'organiser pour mieux s'intégrer dans le processus de gouvernance territoriale en cours avec la décentralisation? Les organisations professionnelles d'éleveurs et d'agriculteurs ainsi que les associations émanant de la société civile peuvent-elles contribuer à réduire ce climat d'insécurité? Dans une première partie, nous expliciterons les facteurs de fragilité qui sont à l'origine de ce contexte d'insécurité plurielle en considérant les principaux groupes socioprofessionnels en milieu rural et les décideurs de cette région. Dans une seconde partie, l'analyse des formes d’insécurité et leurs conséquences sur les systèmes de production concernera spécifiquement la communauté des éleveurs Mbororo de la plaine de la Bénoué (province du Nord). Ensuite, nous discuterons de quelques pistes d'intervention visant la réduction de l'insécurité dans cette région.

\section{Méthodologie}

La compréhension des insécurités qui affectent les populations rurales de cette région s'appuie sur une revue de littérature (Roupsard 1987 ; Seignobos et Weber 2002 ; Levrat, 2010) et sur la connaissance des systèmes de production et du fonctionnement des sociétés rurales que nous avons acquise dans le cadre de différents projets de recherche (Dugué et al., 2006; Kossoumna Liba’a et Havard, 2006; Kossoumna Liba’a, 2008). La compréhension des formes d'insécurité qui touchent les éleveurs Mbororo et l'évaluation de leurs impacts découlent des observations directes ainsi que des enquêtes et entretiens menés dans deux villages Mbororo Djaafun de la plaine de la Bénoué, dans la province du Nord: Ndiam Baba et Laïndé Ngobara (Kossoumna Liba’a, 2008) (figure 1). Ces deux villages constituent le territoire d'attache de ces familles d'éleveurs, qui leur a été affecté par l'autorité coutumière avec l'accord de l'administration. Les familles y ont installé leurs habitats sédentarisés et leurs champs de céréales, mais leurs activités pastorales se déroulent principalement sur des territoires de proximité et de transhumance (Dongmo, 2009). Les enquêtes et observations sur l'évolution des modes de vie et d'activité des éleveurs en rapport avec les insécurités foncières et économiques ont été réalisées en 2006 et 2007 auprès des 36 chefs d'exploitation à Laïndé Ngobara et 28 à Ndiam Baba. Un suivi des activités agricoles et pastorales a été réalisé sur un cycle annuel de mai 2006 à avril 2007.

Les enquêtes explicitant l'importance et les conséquences des prises d'otages ont été réalisées en 2007 auprès des familles d'éleveurs de ces deux villages ayant subi des agressions. Elles ont porté sur la fréquence des prises d'otage au sein des deux communautés, les lieux à risque, les modalités de paiement des rançons et les conséquences de ces agressions sur le mode de vie, les activités et l'avenir de ces éleveurs dans la zone. Pour mener à bien les enquêtes, il a fallu au préalable identifier les lieux de repli des familles après les agressions, dans la mesure où elles avaient toutes quitté leur territoire d'attache. Les résultats ont été confrontés avec les constats d'agressions établis par les services publics de la province du Nord. 
Figure 1 Localisation des villages d'étude dans la plaine de la Bénoué, province du Nord (Cameroun)

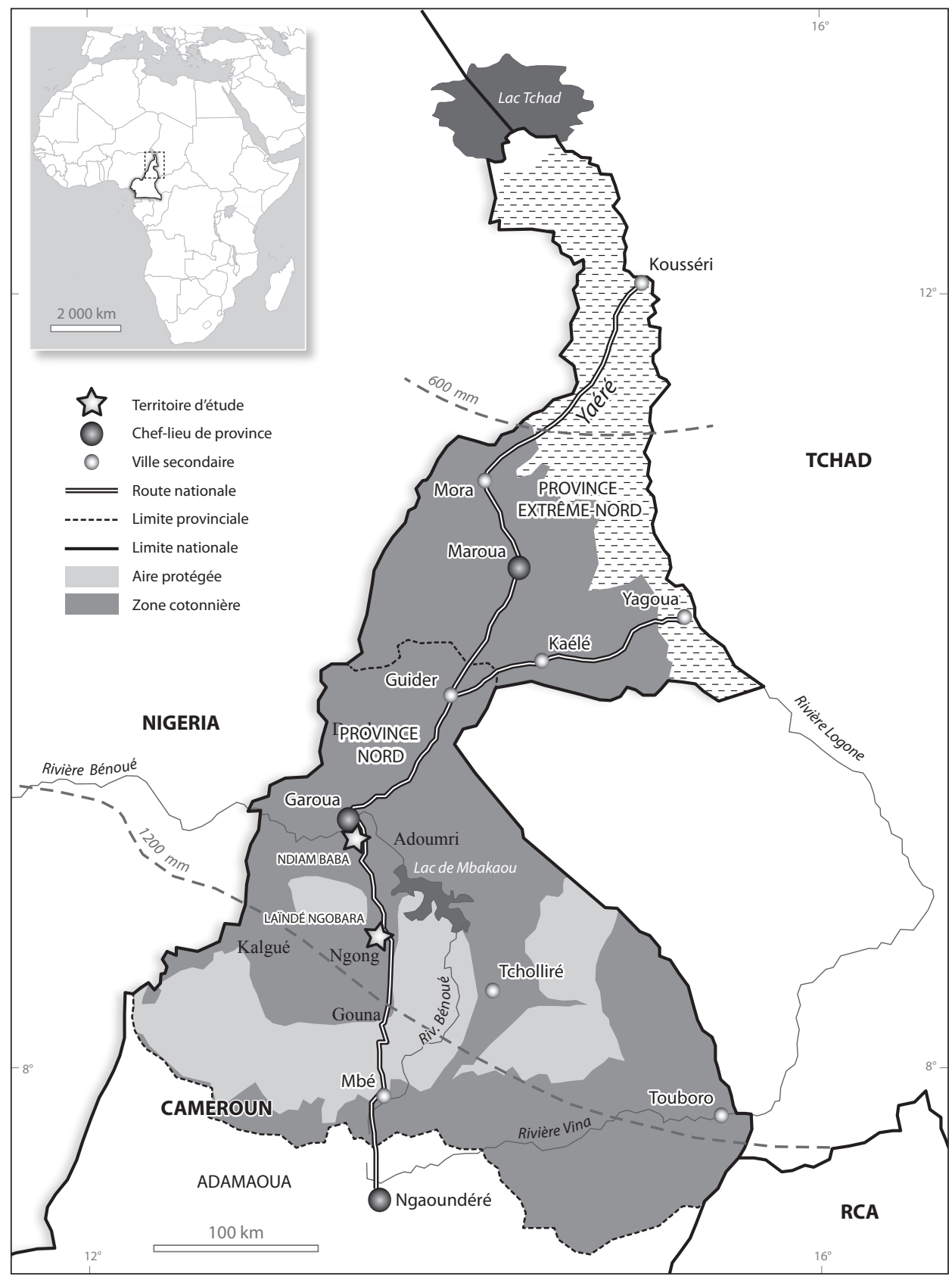




\section{Cadre conceptuel}

Pour analyser les insécurités subies par les éleveurs Mbororo du nord du Cameroun, nous mobilisons le concept de sécurité humaine (Rioux, 2001). Ce concept complète l'approche classique de la sécurité nationale liée aux conflits inter-États ou internes aux pays (coup d’État, guérilla). Il implique de prendre en compte une large gamme de menaces sur les hommes et de mettre l'accent non plus sur l'État face à ses ennemis, mais sur la sécurité des individus et des groupes sociaux (en particulier les plus vulnérables). Selon Rioux (2001), l'absence de sécurité humaine se définit «comme l'ensemble des menaces politiques, économiques, sociales, environnementales et culturelles qui affectent les individus dans leur vie quotidienne et la satisfaction de leurs besoins de base et leur épanouissement». Le débat sur le contenu de ce concept porte sur la distinction entre une interprétation basée uniquement sur les aspects de la violence (freedom from fear) et un cadre d'analyse plus large qui tient compte des aspects du développement humain (freedom from want). La première interprétation, dite «étroite», met l'accent sur le fait que pour que les individus soient en sécurité, ils doivent vivre à l'abri de toute menace omniprésente, violente ou larvée, grâce au droit (lois, règles) et à l'intervention des services publics de sécurité. La seconde interprétation de la sécurité humaine (école du cadre élargi) se réfère à la sécurité de la communauté ou du groupe social, quelle que soit sa taille. Dans ce cas, la sécurité humaine prend en compte un nombre plus important de menaces, allant des menaces traditionnelles déjà évoquées aux menaces liées au développement économique et aux activités humaines comme la pauvreté, l'absence de sécurité sanitaire ainsi que, dans les cas extrêmes, la faim et la famine; les phénomènes accidentels ou continus de pollution et de dégradation des ressources naturelles (désertification) ; les menaces de répression pour des raisons d'opposition à un pouvoir centralisé et abusif; les troubles sociaux et les conflits au sein des communautés et entre elles. Pour analyser les insécurités chez les éleveurs Mbororo au nord du Cameroun, nous retiendrons cette conception «large» de la sécurité humaine (Ibid.).

Les recherches sur les causes et la durée des faits de violence en Afrique subsaharienne menées par Collier et Hoeffler (2000) enrichissent ce cadre conceptuel. Elles sont marquées par le débat entre deux écoles de pensée: greed contre grievance (cupidité contre frustration) (Jozan et Ray, 2009). Dans le premier cas, les causes et l'entretien des violences sont d'ordre économique: les fauteurs de troubles profitent de ces situations insécurisantes et violentes pour capter la rente engendrée par les conflits (prélèvements auprès des populations, trafics et commerces illicites). Dans le second cas, les frustrations vécues par certaines catégories de la population sont à l'origine des violences et des conflits; ce peut être le cas de groupes vivant dans une extrême pauvreté en ville ou ayant perdu certains droits d'accès à des ressources naturelles dans les campagnes.

La seconde catégorie de concepts mobilisée dans cet article se rapporte à la participation des citoyens à la gestion du territoire. Cette question amène à mieux comprendre les relations, d'une part, entre les différents groupes socioprofessionnels du fait des asymétries de pouvoir et, d'autre part, entre les populations rurales et l'État. Les travaux de Hyden (1983) et Geschiere (1984) mettent en évidence différents types de relation entre l'État et les paysanneries en fonction du niveau d'insertion des populations rurales dans la vie économique (insertion au marché, contractualisation 
avec l'agroindustrie, etc.) ainsi que dans le jeu politique (capacités d'intervention et de négociation des organisations professionnelles, implication des ruraux dans la gestion des collectivités locales). L'analyse des relations de pouvoir entre les citoyens et l'État a été développée par Foucault (1992) qui part du fait que «les relations de pouvoir s'enracinent dans l'ensemble du réseau social et ne peuvent se réduire aux institutions fussent-elles politiques». L’auteur précise que «les formes et les lieux de "gouvernement" des hommes les uns par les autres sont multiples dans une société; ils se superposent, s'entrecroisent, se limitent et s'annulent parfois, se renforcent dans d'autres cas». Cette conception du pouvoir semble correspondre aux réalités des sociétés africaines contemporaines sur lesquelles s'est plaqué 1'État postcolonial. Cette superposition de pouvoirs constitue-t-elle un atout ou une contrainte pour assurer la sécurité individuelle et collective dont les populations africaines ont besoin? Ainsi, Le Roy et Trotha (1993) ont mis en évidence des dispositifs de réduction des insécurités et de leurs conséquences en considérant que les sociétés africaines ont dû inventer «des dispositifs du quotidien qui n’annulent pas la violence mais la mettent à distance». Ces dispositifs non institutionnalisés compensent par exemple les carences des systèmes publics. I1s s'adaptent aux nouveaux besoins et collectent des fonds en mobilisant les ressortissants de ces régions installés dans les villes et qui peuvent aider leur village d'origine. La particularité de l'informel est de combiner les besoins avec les valeurs, les croyances, les règles et pratiques de solidarité communautaire, ces dernières étant fondées sur la continuité de l'échange (Le Roy, 1993).

Les enseignements des travaux sur les relations entre État et paysanneries nous permettent de mieux comprendre les différentes formes d'insécurité qui prévalent au nord du Cameroun. Ils seront mobilisés en fin d'article pour aborder quelques pistes de résolution de ces situations complexes.

\section{Le nord du Cameroun: une région en crise}

\section{Un pouvoir coutumier toujours présent}

Les évolutions des sociétés agraires et pastorales au nord du Cameroun ces 30 dernières années sont marquées par l'origine du peuplement de cette région. Au début du XIXe siècle, les Peul Fulbe venus de l'ouest ont conquis les plaines du Cameroun septentrional grâce à leur organisation militaire et leur cavalerie, rejetant une bonne partie des populations autochtones dans les massifs montagneux périphériques (Boutrais, 1984; Beauvillain, 1989). Ils ont ainsi acquis, dans ces plaines, le droit de gestion du foncier agricole et pastoral.

L'organisation sociale traditionnelle dans cette région est encore aujourd'hui structurée autour d'une autorité supérieure d'ethnie Peul Fulbe, le laamii'do (pluriel lamibé) qui est à la tête du territoire qu'il administre, le lamidat (Ndembou, 1998). Le territoire est divisé en ar'do regroupant plusieurs villages. Le village (wuro) est la plus petite unité administrative traditionnelle commandée par le jawro, l'équivalent du chef de village des autres régions d'Afrique centrale. Le peuplement de ces lamidat est dominé par des ruraux: des agriculteurs s'adonnant aux productions vivrières et à la culture du cotonnier, et des éleveurs (Peuls Fulbe et Mbororo) pratiquant l'élevage extensif de ruminants. Malgré l'importance démographique des populations agricoles non 
Fulbe, le découpage territorial issu de la conquête peule persiste aujourd'hui ainsi que les prérogatives des lamibé en particulier pour la gestion du foncier agricole et pastoral. Ainsi, même si la législation foncière camerounaise existe depuis 1932, elle n'empêche pas la prédominance du droit coutumier sur le droit «moderne» en zone rurale: «La terre appartient au laamii'do qui en concède l'usage à ses sujets à condition qu'ils paient la zakkat» (Beauvillain, 1989). Concrètement, les agriculteurs migrants doivent obtenir l'autorisation du représentant du laamii'do pour défricher une parcelle ou obtenir le droit d'usufruit d'une parcelle laissée par un tiers. En plus de la gestion du foncier rural, le laamii'do et les chefs sous son autorité gèrent la circulation du bétail sur le territoire du lamidat. Dans certains cas, ils prélèvent des taxes sur le commerce des produits vivriers (céréales, arachide) et collectent l'impôt traditionnel sur les revenus des ruraux, la zakkat ${ }^{2}$ (Ibid.). En contrepartie, le laamii'do doit apporter secours aux plus démunis et aux populations touchées par des accidents climatiques ou des incendies. Plus globalement, il doit faire régner la paix et la bonne entente entre les différents groupes sociaux qui peuplent son lamidat. Cette capacité de contrôler la totalité de son territoire (les activités, l'accès aux ressources naturelles et les flux de biens et de personnes) au besoin en mobilisant ses gardes (daugari), constitue la principale force du laamii'do.

Aujourd'hui, le système traditionnel de gouvernance des lamidat cohabite avec les structures et règles de gestion étatiques modernes apparues lors du protectorat français et renforcées par l’État camerounais après l'indépendance au niveau de chaque province (gouverneur, préfets et sous-préfets coordonnant les services publics).

\section{Pression démographique, raréfaction des ressources naturelles et aléas pluviométriques}

La croissance démographique et l'ouverture du nord du Cameroun aux marchés régionaux et internationaux ont fortement modifié le fonctionnement des sociétés rurales (Roupsard, 1987). La croissance démographique annuelle en milieu rural entre 1980 et 2000 est estimée pour la province du Nord à 6\%: $3 \%$ environ d'augmentation naturelle et l'équivalent lié aux flux migratoires des zones les plus peuplées de l'extrême-nord vers la plaine de la Bénoué (figure 1). Ces migrations s'expliquaient par la forte disponibilité en terres agricoles dans la province du Nord à cette période. Le développement de la production cotonnière et surtout de la culture attelée, promues par la Société de développement de la culture cotonnière du Cameroun (SODECOTON), ont contribué à augmenter la surface cultivée par actif agricole. Durant la même période, la densité en bovins détenus par les Peuls s'est accrue du fait du croît naturel, mais aussi d'un flux migratoire d'éleveurs Mbororo venant du Nigeria. En même temps, l'État camerounais a augmenté la surface classée en aires protégées de préservation de la faune sauvage dans la province du Nord, qui passe de $30 \%$ de la superficie provinciale en 1969 à $46 \%$ en 2002.

La densité de population rurale de la province dépasse 50 hab. $/ \mathrm{km}^{2}$, avec certaines zones à plus de 100 hab. $/ \mathrm{km}^{2}$ (Dongmo, 2009). Ces évolutions ont entraîné une forte concurrence entre ces différents acteurs pour l'accès aux ressources naturelles. La culture continue sans pratique de la jachère s'est généralisée. Du fait d’un accroisse-

2 De l'arabe Zakkat, «aumône instituée par le Coran». 
ment du cheptel bovin et des défrichements non contrôlés réalisés par les agriculteurs, les parcours et pistes à bétail sont devenus trop exigus. Leur accès est rendu difficile pour les troupeaux de grande taille. La fréquence des conflits fonciers et des dégâts occasionnés aux cultures par les troupeaux augmente. Mais les populations rurales sont surtout confrontées aujourd'hui à une forte dégradation des terres agricoles et des parcours (baisse de fertilité du sol, prolifération d'adventices et de plantes non prisées par le bétail). Cette dégradation est la principale cause de la paupérisation des agriculteurs qui ont du mal à faire face aux besoins de leurs familles et encore plus à l'entretien de leurs outils de production (renouvellement du matériel et du cheptel de trait, et entretien de la fertilité des sols).

Du fait de ces évolutions, l'insécurité foncière, tant pour les agriculteurs que pour les éleveurs, constitue une entrave majeure aux politiques de développement et de protection de l'environnement dans le nord du Cameroun. La question foncière s'est donc imposée d'elle-même dans la région et a été progressivement intégrée dans les projets de développement qui ont essayé de la résoudre mais avec peu de succès. Aujourd'hui, elle a été momentanément délaissée parce qu'elle est complexe et que les projets n'ont pas encore trouvé de solution simple pour la résoudre: les accords locaux entre acteurs ont beaucoup de mal à se maintenir après l'arrêt des projets (Raimond et al., 2010).

Enfin, la fréquence des aléas pluviométriques a augmenté depuis une vingtaine d'années (M’Biandoun et al., 2003). Les pluies sont plus souvent mal réparties en début de campagne agricole (mai, juin), période des labours et des semis, le report des semis en juillet entraînant une baisse de rendement des cultures. La fréquence des excès de précipitations au mois d'août et au début septembre entraîne de plus en plus souvent des destructions de cultures dans les bas de toposéquence.

\section{Une crise économique qui perdure}

Au nord du Cameroun, la crise économique a d'abord été urbaine à la suite d'une baisse de moitié des salaires des fonctionnaires en 1992 et de la dévaluation du FCFA en 1994 (Courade, 2000). La demande en biens de consommation des urbains est alors réduite, tandis que le tissu commercial et industriel émergeant périclite, sauf les entreprises en lien direct avec la filière coton qui bénéficie, elle, de la dévaluation du FCFA. Pour les agriculteurs, la baisse des revenus agricoles est due en premier lieu à la dégradation de la fertilité des sols, donc des rendements. De 1992 à 2000, la baisse de rendement en coton-graine est évaluée à 1,7\% par an (Abbou Abba et al., 2006) alors que la filière cotonnière est considérée comme le moteur du développement régional et un facteur de stabilité de cette région, du fait des revenus et des services qu'elle procure (appui à l'équipement des exploitations, entretien des pistes rurales) (Deveze, 2006).

La baisse du revenu agricole s'accentue à partir des années 2000 du fait d'abord de l'augmentation du prix des engrais minéraux, de la stagnation et de la baisse, certaines années, du prix d'achat du coton-graine au producteur et, enfin, de la réduction de la surface de culture du coton (Kossoumna et Havard, 2006; Levrat, 2010). La production de coton-graine, qui était de 300000 tonnes en 2004, passe à 125000 tonnes en 2009 . De plus, les producteurs sont peu organisés dans le secteur des productions vivrières 
et doivent faire face à des commerçants qui n’hésitent pas à spéculer sur ces produits de base dont une partie est exportée vers les grandes villes du sud. Dans ce contexte de crise économique, les éleveurs s'en sortent mieux, car ils achètent peu d'intrants et peuvent vendre leurs animaux sur les marchés du Nigéria où la demande reste forte. La crise de la filière coton et la mévente, certaines années, des productions vivrières ont des répercussions graves sur les acteurs en présence. Dans la région, on observe une dégradation de la santé et de la scolarisation des populations rurales ainsi qu'une aggravation de la corruption et de la délinquance. Face à cette crise socioéconomique, l'État a surtout réagi dans le domaine de la production agricole en subventionnant les engrais depuis 2009.

\section{Un climat grandissant de violence et d'insécurité}

La violence a toujours été présente dans cette province du Cameroun, en particulier du fait des pratiques autoritaires de certains chefs coutumiers (bastonnade, emprisonnement dans les geôles des Lamibé). Malgré cela, un relatif climat de sécurité y régnait des années 1960 à 1980, dans la mesure où les conflits entre ruraux (agriculteurs, éleveurs, gestionnaires des terres, usufruitiers) étaient réglés assez rapidement par ces autorités coutumières ou par l'administration. Le climat d’insécurité apparaît d'abord en milieu urbain par la recrudescence des vols et des attaques à main armée, à partir des années 1990. À la même époque, le phénomène des «coupeurs de route» se développe le long des grands axes routiers du Cameroun septentrional. Ces bandits armés attaquent surtout les commerçants transportant des fonds. Ils s'en prennent aussi aux villageois après le paiement du coton. Progressivement, les commerçants et la filière coton s'organisent pour faire face à ces agressions : convois encadrés par les forces de l'ordre, informatisation des transferts de fonds. Ceci peut expliquer le développement du phénomène des prises d'otages en milieu rural à partir de 2003, qui concerne presque exclusivement les communautés d'éleveurs Mbororo. Des groupes de 20 à 30 hommes armés et très organisés font irruption dans les campements d'éleveurs et enlèvent les jeunes enfants. En échange de leurs otages, les bandits réclament de fortes sommes d'argent.

L'insécurité en milieu rural dans cette région est en fait plus complexe et ne peut pas se résumer aux agressions physiques et aux vols, même si ces faits sont les plus graves et doivent être combattus en priorité. Pour un agriculteur et sa famille ne disposant que de deux à trois hectares de mauvaises terres, l'insécurité est d'ordre alimentaire et foncier. Sans moyens, il ne peut pas cultiver plus de terre même s'il dispose d'une grande famille: la terre peut se louer et même de façon officieuse s'acheter, mais cela implique de mobiliser des fonds et un bon réseau de connaissances pour pouvoir négocier avec les autorités coutumières, gestionnaires des terres. Même si cet agriculteur trouvait des fonds, il hésiterait à investir dans l'aménagement et la restauration de la fertilité de ses parcelles en vue d'améliorer sa production, car il ne dispose pas d'un titre foncier. Il est soit simplement usufruitier de la terre que lui ou ses ascendants ont défrichée, soit locataire de parcelles sans bail et donc sans garantie de la cultiver dans la durée. Après l'amélioration des conditions de production (fumure organique, aménagement antiérosif), les gestionnaires des terres sont tentés de récupérer les parcelles mises en location pour les cultiver eux-mêmes ou les faire cultiver par leurs proches. 
De plus, cet agriculteur doit faire face, depuis une dizaine d'années, à une plus grande fréquence des risques climatiques, comme nous l'avons mentionné. Les baisses de production peuvent être importantes en cas de sécheresse en fin de cycle des cultures, de retard de semis ou d'inondations. Cette situation aléatoire n'incite pas les agriculteurs à intensifier leurs systèmes de culture en vue d'accroître la production.

Ces diverses formes d'insécurité touchent la majorité des familles rurales du nord du Cameroun. Cette combinaison de formes d'insécurité sera explicitée plus précisément dans le cas des éleveurs Mbororo, dans la partie suivante.

\section{Impacts des insécurités sur les communautés d'éleveurs Mbororo}

\section{Histoire, sédentarisation et modifications des pratiques d'élevage}

Pour comprendre l'origine des diverses formes d'insécurité touchant les éleveurs Mbororo, il est nécessaire d'aborder leur histoire récente et les raisons qui les ont amenés à se sédentariser. Les communautés Mbororo se sont installées au nord du Cameroun au cours du XXe siècle en provenance du Niger et du Nigéria, à la recherche de zones peu peuplées et riches en pâturage. Ils ont obtenu, dans le bassin de la Bénoué, des droits de pâture de la part des autorités coutumières, les lamibé Peul Fulbe. Afin de renforcer leurs droits fonciers et leurs positions politiques, les Mbororo, initialement nomades, ont opté depuis une vingtaine d'années pour la sédentarisation de leur habitat. Leurs campements ont été progressivement reconnus par l'État comme des villages, et ils ont obtenu des cartes d'identité et le droit de vote au Cameroun. Cette fixation s'explique par leur souci d'améliorer leurs conditions de vie: accès à l'eau par des forages et puits, école et centre de santé au village ou à proximité. Elle facilite aussi l'accès aux informations utiles au commerce du bétail et donc au développement économique des unités de production. Grâce à la fumure animale, la sédentarisation s'accompagne du développement, sur de petits espaces proches des habitations, de la culture continue et intensive du maïs. La majorité des éleveurs sédentarisés assurent ainsi la sécurité alimentaire de leur famille (Kossoumna Liba’a et al., 2010).

Dans ces conditions, les projets de développement rural considèrent aujourd'hui ces communautés sédentarisées comme des partenaires potentiels, surtout si elles acceptent de créer des organisations ou groupements d'éleveurs. Des projets de développement et les services techniques ont œuvré pour faire classer, dans les territoires d'attache, des espaces exclusivement pastoraux appelés "parcours sécurisés bornés ». Dans le cas des deux communautés étudiées, la superficie totale allouée par village ne dépasse pas 200 ha, dont 150 ha environ classés en parcours sécurisé. Ce petit espace peut alimenter au mieux 200 bovins toute l'année si on y ajoute la production de paille des 50 ha de céréales (Ibid.). Mais les effectifs bovins de ces deux communautés varient selon les années entre 1000 et 1200 têtes. De ce fait, les éleveurs sont obligés de maintenir la pratique de la transhumance, mais les déplacements des troupeaux sont rendus difficiles par le rétrécissement et parfois la disparition des pistes à bétail (burtol). Le plus souvent, les bergers empruntent les grandes pistes rurales et les bords des routes goudronnées, dès que les champs sont emblavés. De plus, le renforcement des aléas climatiques (arrivée tardive ou précoce des pluies), l’insécurité et les conflits avec les agriculteurs amènent les éleveurs à changer de stratégie de transhumance, 
parfois chaque année, même si les lieux d'accueil restent généralement les mêmes. Les éleveurs adoptent de plus en plus des stratégies individuelles pour accéder aux espaces de pâturage, dont la surface diminue progressivement. On assiste donc à un amenuisement des décisions collectives relatives à l'organisation des transhumances à cause de la compétition entre éleveurs pour l'accès aux espaces de pâturage. La pratique de l'agriculture avec le souci de fertiliser les parcelles a aussi amené les éleveurs à modifier l'organisation de leur travail. L'éleveur, chef d'exploitation, souhaite en effet garder un œil sur ses champs. Le développement des transports motorisés et de la téléphonie mobile lui permet de se rendre rapidement, si besoin, sur les lieux de transhumance. Même si l'élevage reste au centre des activités des éleveurs, la sédentarisation les pousse à s'occuper différemment du bétail. Le troupeau est de plus en plus confié à des salariés pour, d'une part, maximiser l'accès aux divers espaces de pâturage morcelés en scindant le grand troupeau en sous-unités et, d'autre part, pour scolariser les enfants Mbororo traditionnellement affectés au gardiennage (Kossoumna Liba’a, 2008).

La sédentarisation a bien rapproché ces éleveurs des autorités administratives et des projets de développement, mais cela n’a pas été suffisant pour les insérer dans le jeu politique, économique et social local et pour garantir leur sécurité. Avec la nationalité camerounaise, les éleveurs Mbororo peuvent se faire représenter dans les instances locales de décision comme les communes rurales ou dans les partis politiques. Mais leur poids dans ces instances est quasiment nul du fait, entre autres, de leur analphabétisme. De plus, les Mbororo ont peu misé dans le passé sur l'éducation de leurs enfants qui sont très peu présents dans l'administration et les services techniques.

\section{Insécurités multiformes et fragilisation des systèmes de production}

\section{Insécurité foncière}

Les projets de sécurisation des parcours par le bornage n’ont pas donné les résultats escomptés, la réalisation de ce bornage n’ayant pas toujours associé toutes les parties prenantes. Par exemple, dans le territoire de Laïnde Ngobara, quelques parcelles de culture existaient dans la zone pastorale avant son bornage et les agriculteurs des villages voisins n’ont pas été indemnisés ni même associés à cette zone. Ils ont continué d'y cultiver, rendant ainsi difficile la valorisation de ce parcours en saison de culture. Les accords locaux obtenus grâce à l'intermédiation des projets ont bien souvent du mal à se maintenir dans la durée. Plus globalement, les éleveurs reprochent aux autorités coutumières de ne pas faire respecter les accords obtenus de longue date et le plus souvent actés par l'administration, comme la délimitation des pistes à bétail (burtol) et des grands parcours (hurum).

Pourtant, la loi de 1976 sur la gestion du domaine national a renforcé l'arsenal juridique ainsi que le pouvoir des services publics pour régler les conflits et organiser l'occupation des terres. Mais aujourd'hui, les autorités publiques n'assument plus la fonction d'arbitrage et de régulation. Les mécanismes de résolution des conflits entre agriculteurs et éleveurs ne fonctionnent pas bien faute, entre autres, de moyens logistiques. Les services techniques ne sont plus en mesure de contrôler l'intégrité des pistes à bétail et d'éviter les défrichements des agriculteurs dans certaines zones pastorales. Dans ce contexte, les éleveurs adoptent de plus en plus des stratégies 
individuelles pour accéder aux petits espaces de pâturage résiduels en scindant le troupeau en petites unités (Dongmo, 2009). Ainsi, la gestion de l'espace est soit délaissée, soit mise en œuvre de manière arbitraire sans prise en compte objective des besoins des différents groupes socioprofessionnels, comme c'est le cas dans plusieurs lamidat de la province du Nord (Seignobos, 2010). Les autorités coutumières n’hésitent pas, parfois, à brader les zones de parcours au profit des agriculteurs et des citadins investissant dans l'agriculture et avec lesquels ils monnayent le droit de défricher et de mettre en culture.

La diminution des surfaces pâturables et des pistes à bétail fragilisent ces systèmes pastoraux reposant sur la mobilité du bétail et les parcours naturels collectifs. Cette insécurité foncière n'encourage pas les éleveurs à s'investir dans la gestion des ressources pastorales en pratiquant, par exemple, la mise en défens des parcours dégradés ou leur ensemencement avec des espèces fourragères améliorantes ou, encore, le contrôle de l'émondage des arbres fourragers. Les éleveurs considèrent qu'ils n'ont pas la garantie de récupérer les fruits d'un tel investissement (Dongmo, 2009).

\section{Insécurité fiscale et insécurité juridique}

L'insécurité fiscale est liée au fait que les éleveurs sont confrontés à diverses formes de taxation venant de l'État, des communes rurales et des lamidat, dont l'importance varie au fil des ans. Les taxes traditionnelles sont diverses et le montant est souvent calculé de façon arbitraire. Celle qui est versée au laamii'do par les éleveurs est appelée jomorgol laamii'do ou hacce lesdi (droit d'accès à la terre cultivable et au parcours). La zakkat, impôt sur la production agricole, est versée le plus souvent en nature au laamii'do sous la forme de sacs de céréales. Les éleveurs, comme les autres ruraux, s'acquittent aussi régulièrement des collectes ponctuelles (umroore laamii'do) qu'impose le laamii'do aux habitants de son territoire lors d'un événement important (funérailles, fête, réception d'une autorité). Les éleveurs n’ont pas d'autre choix que d'acquitter ces taxes, car le non-payeur est passible d'expulsion du lamidat.

Sans prendre un caractère ouvert, les conflits entre éleveurs et autorités traditionnelles sont fréquents. Les éleveurs contestent le montant des amendes qu'on leur impose à la suite de dégâts causés par leurs troupeaux aux cultures des agriculteurs. Ainsi, le sentiment d'une certaine complicité entre les chefs coutumiers et les agriculteurs au détriment des éleveurs Mbororo prévaut chez ces derniers. Mais ce parti pris peut s'expliquer par le fait que les éleveurs disposent d'un capital - le bétail - d'une valeur bien supérieure à celui des agriculteurs et facilement monnayable. De ce fait, pour qui veut s'enrichir, il est préférable de taxer les éleveurs, car ils ont les moyens de payer.

\section{Insécurité physique et impacts sur les pratiques des éleveurs Mbororo}

Le phénomène de rapt des enfants Mbororo est apparu récemment, au début des années 2000, et a pris une grande ampleur en 2007 malgré les interventions des forces de l'ordre (Saïbou, 2006; Daïrou, 2007). Bien que proche de la ville de Garoua où se concentrent les services de sécurité, le département de la Bénoué, cadre de notre étude, est aussi concerné par ce phénomène (tableau 1). Les kidnappeurs ont pris conscience que la vie d'un enfant est un moyen de chantage très efficace pour amener les éleveurs à vendre tout ou une partie de leur troupeau. En 2007 à Laïndé Ngobara, huit familles ont dû vendre au total 23 bœufs pour un montant de 4600000 FCFA pour 
payer la rançon exigée pour huit enfants. La communauté Mbororo de la province du Nord a entrepris des démarches pour saisir les autorités administratives et assister les familles d'éleveurs victimes des exactions commises en 2007. Les interventions de certaines associations d'éleveurs (comme Hoore Pulaaku) pour limiter les attaques sont rendues difficiles par le fait que certains membres des groupes armés ${ }^{3}$ qui ont été appréhendés étaient des jeunes Mbororo issus des communautés d'éleveurs installées dans la province. Ces jeunes fournissent les renseignements utiles aux groupes: la localisation du bétail, les ventes d'animaux et les noms des familles les mieux dotées.

\section{Tableau 1 Préjudices causés aux éleveurs de la province du Nord (Cameroun) entre janvier et mai 2007}

\begin{tabular}{l|c|c|c|c}
\hline Département & $\begin{array}{c}\text { Personnes } \\
\text { tuées }\end{array}$ & $\begin{array}{c}\text { Personnes } \\
\text { kidnappées }\end{array}$ & $\begin{array}{c}\text { Bovins } \\
\text { vendus }\end{array}$ & $\begin{array}{c}\text { Montant des rançons } \\
\text { payées en FCFA }\end{array}$ \\
\hline Bénoué & 9 & 179 & 26817 & 341610500 \\
Mayo Rey & 134 & 175 & 73200 & 852285000 \\
Mayo Louti & 0 & 0 & 10000 & 150000000 \\
Faro & 0 & 0 & 0 & 0 \\
Total province du Nord & $\mathbf{1 4 3}$ & $\mathbf{3 5 4}$ & $\mathbf{1 1 0 0 1 7}$ & $\mathbf{1 3 4 3 \mathbf { 8 9 5 } 5 0 0}$ \\
\hline
\end{tabular}

Source: Dairou D., 2007.

À la suite de ce type d'agressions, les villages d'éleveurs Mbororo étudiés se sont vidés de leurs habitants entre juillet et septembre 2007. Les familles se sont refugiées dans les bourgs peuplés principalement par des agriculteurs et commerçants, au bord des grandes routes considérées plus sûres. Une partie du bétail des éleveurs de Laïndé Ngobara a été transférée au Nigeria. Mais ces familles se sentent aujourd'hui camerounaises ou, du moins, souhaitent rester à proximité de leur territoire d'attache, qu'elles considèrent comme leur bien et qui fournit toujours une bonne partie de leur alimentation. Les champs de céréales se sont maintenus dans ces territoires malgré le départ de la majorité de la population, entraînant pour celle-ci des déplacements longs ou coûteux.

Dans ce contexte d'insécurité, les éleveurs ont dû faire évoluer leurs pratiques et la conduite de leurs troupeaux. Si auparavant ils exhibaient fièrement leur bétail, ils sont aujourd'hui obligés de scinder les grands troupeaux en sous-unités de 20 à 30 têtes afin de limiter les risques de vol, mais surtout de cacher l'importance de leur capital. Ce fractionement forcé entraîne un accroissement du coût du gardiennage, du fait du recours à plusieurs bergers salariés. Ce climat d'insécurité a amené les citadins propriétaires de bovin à moins confier leurs animaux par contrat aux Mbororo à cause des risques encourus. Cette pratique permettait aux éleveurs les plus pauvres d'avoir une source de revenu régulière et de reconstituer un troupeau en gardant un veau mâle sur deux nés. Les Mbororo sans bétail, restés aujourd’hui dans la région et employés auparavant comme bergers, se retrouvent fréquemment sans emploi, et leur reconversion est difficile. La mendicité et l'alcoolisme pour les hommes, parfois la prostitution pour les femmes, sont apparus. N'ayant pas de perspectives

3 Parmi les bandits arrêtés au Cameroun, on trouve des Nigérians, des Camerounais, des Tchadiens et des Centrafricains d'origines socioprofessionnelles diverses. 
pour reconstituer un troupeau, certains de ces Mbororo s'associent à des groupes de malfaiteurs. Pillés et rançonnés, des anciens éleveurs deviennent parfois eux-mêmes les agresseurs de leurs semblables.

\section{Réduire les insécurités au nord du Cameroun: une équation complexe}

Nous ne traiterons pas, dans cette discussion, du rôle régalien de l'État comme garant de l'intégrité des biens et des personnes. L'analyse du fonctionnement des services publics nécessiterait de développer des enquêtes spécifiques, ce qui sort du cadre de notre étude. Nous nous focaliserons ici sur le renforcement des capacités des acteurs ruraux à dialoguer avec les autres acteurs, vu comme un des moyens pour réduire les insécurités.

\section{Faire vivre ensemble sur un même territoire une diversité de communautés}

Malgré l’arsenal législatif, l'État n’a pas réussi au nord du Cameroun à imposer la loi foncière nationale ni à éliminer les droits fonciers traditionnels. L'accès à la terre agricole et pastorale demeure contrôlé par la chefferie coutumière, qui n'accorde aux producteurs que des droits précaires. Depuis qu'elles ont été impliquées dans le jeu politique local et national, ces chefferies ont confirmé leur rôle central dans la gestion du foncier. Concernant les agriculteurs, différents projets et structures de développement leur ont permis de s'organiser, de développer de nouvelles activités (par exemple l'extension de la culture de l'oignon) et d'accéder à des services, en particulier dans le cadre la filière coton (Devèze, 2006). Mais l'insécurité foncière constitue toujours un frein à l'adoption des innovations agricoles et pastorales, qui implique un investissement de base à valoriser et rentabiliser à moyen terme: les aménagements antiérosifs et agroforestiers, ainsi que la couverture permanente du sol par les résidus de culture pour en améliorer la fertilité et l'amélioration des parcours. Aujourd'hui, l'expérimentation de règles innovantes de gestion du foncier (baux pluriannuels) se heurte à l'immobilisme des autorités coutumières et au faible engagement de l’État (Seignobos, 2010). Ces expérimentations sont menées dans le cadre de projets à durée déterminée et les accords locaux ont beaucoup de mal à se maintenir ensuite dans un cadre institutionnel permanent comme celui des communes rurales (Raimond et al., 2010).

Concernant la place de l'élevage dans l'espace régional, l'applicabilité des accords locaux relatifs aux espaces de pâturage doit être analysée au cas par cas. La sédentarisation des familles d'éleveurs n'a pas modifié en profondeur les pratiques d'élevage, basées sur la mobilité des troupeaux et la transhumance. Vu la superficie des territoires d'attache qui leurs sont alloués, ces éleveurs ne peuvent pas opter pour un modèle techniciste de sédentarisation de l'élevage fondé sur des cultures fourragères et des parcours améliorés, comme cela est souvent préconisé par les services publics. Le maintien de l'élevage au niveau régional et, par conséquent, de l'approvisionnement en produits animaux pour les populations qui y vivent vont donc dépendre de la capacité des acteurs à préserver les grands parcours de transhumance ou de proximité et leurs voies d'accès. Mais les règles de vaine pâture devront évoluer, car les agriculteurs qui produisent de grandes quantités de paille de céréales commencent à développer 
leurs propres élevages de ruminants ou cherchent à conserver cette biomasse sur le sol pour en améliorer progressivement la fertilité (Naudin et al., 2010). Le partage entre agriculteurs et éleveurs de cette ressource alimentaire essentielle pour le bétail en saison sèche implique d'imaginer des relations contractuelles gagnant-gagnant. Par exemple, les éleveurs peuvent s'engager à ne pas valoriser, en saison sèche, des espaces couverts de résidus de culture. En contrepartie, les agriculteurs leur garantiraient de ne pas morceler les grands parcours et les pistes à bétail. Mais les deux communautés doivent être en mesure de produire plus de biomasse végétale sur les mêmes espaces et donc d'adopter des innovations comme l'association des céréales avec des plantes fourragères (brachiaria, pois d'angole) (Dugué et al., 2011). Ce début d'intensification des systèmes de production par une meilleure gestion de la biomasse végétale concerne l'ensemble des producteurs, mais devra se faire progressivement avec les éleveurs. Pour cela, les communautés d'éleveurs doivent être mieux prises en compte dans les programmes de développement. Des zones cultivables doivent leur être affectées pour accroître la production de biomasse par l'introduction de cultures fourragères, d'abord sur de petites surfaces et avec un dispositif d'appui spécifique complémentaire à celui existant pour les agriculteurs dans le cas de la filière coton. D’une façon plus globale, les éleveurs et leurs organisations devraient être consultés régulièrement pour l'élaboration des politiques d'aménagement du territoire. Par exemple, les éleveurs et les services de l'élevage devraient pouvoir discuter, avec les autres acteurs, du déclassement de certaines aires protégées pauvres en faune sauvage et sans intérêt économique ou environnemental majeur.

\section{Renforcer les capacités des producteurs et de leurs organisations}

La sédentarisation des familles d'éleveurs Mbororo pouvait a priori apparaître comme un facteur favorisant la sécurité de ces populations: proximité des services publics, meilleure insertion dans le jeu sociopolitique communal et provincial et appui par des projets de développement. Il s'avère que, dans le contexte du nord du Cameroun, ces mécanismes n’ont pas fonctionné et les Mbororo constituent toujours un groupe marginalisé et vulnérable. D’une façon plus globale, les ruraux de cette région ont du mal à faire entendre leurs points de vue afin de trouver des appuis pour atténuer les insécurités qu'on a pu caractériser. Pour cela, le renouvellement des relations entre l'État, les autorités coutumières et les paysanneries constitue un enjeu pour le développement de cette province. Il passe en partie par le renforcement des capacités d’intervention collective des ruraux et donc de leurs organisations.

Les associations d'éleveurs, le plus souvent suscitées par l'administration et les projets, sont jeunes et peu formées pour faire face à ces insécurités. Elles manquent de soutien institutionnel et de capital social pour peser sur les décisions concernant l'avenir de l'élevage dans cette région. Elles ont du mal à mettre en place des activités comme du crédit pour l'embouche des ruminants ou l'approvisionnement en produits vétérinaires (Dugué et al., 2008). Les organisations d'éleveurs Mbororo ont comme priorité la reconnaissance de droits d'usage sur des parcours naturels (hurum) afin d'assurer l'alimentation du bétail en saison des pluies. La professionnalisation de ces associations doit d'abord être orientée vers une amélioration de leurs connaissances des lois et règlements et de leurs capacités de négociation. Ainsi, leur intégration et leur contribution aux instances où se discute l'avenir de l'élevage dans cette région en seront accrues. 
Ces associations doivent aussi débattre de l'évolution du métier d'éleveur dans la région. Si les revendications foncières sont légitimes, elles doivent aussi aborder la question de l'intensification d'une partie du cheptel: produire plus sans accroître l'emprise spatiale de l'élevage et en investissant du temps de travail et/ou du capital. La transmission intergénérationnelle des savoirs, des pratiques d'élevage et des règles collectives de gestion des ressources constitue aussi un élément important de la durabilité des systèmes pastoraux. Les fils d'éleveurs sont aujourd'hui moins intéressés par la conduite du troupeau que ceux de la génération précédente. En effet, au fur et à mesure qu'ils se scolarisent et que s'accentuent les contraintes liées à la garde du troupeau, les jeunes se tournent vers d'autres activités comme le commerce et le transport de biens et de personnes. Dans ce cas, le jeune pluriactif recrute un berger pas nécessairement Mbororo pour garder un troupeau résiduel. Ce processus fragilise la transmission des savoirs, le fonctionnement des communautés et des organisations d'éleveurs.

Les organisations d'agriculteurs sont mieux structurées, mais elles dépendent surtout de la culture cotonnière, ce qui les fragilise en cas de crise dans cette filière. Ces organisations pourraient jouer un rôle dans la limitation de certaines formes d'insécurité. Par exemple, en s'engageant dans la commercialisation et le stockage des céréales, elles peuvent peser sur le marché régional en participant à la régulation des prix, gage de sécurité alimentaire pour l'ensemble de la population. Le prix moyen de vente des céréales par les agriculteurs serait plus élevé, et les consommateurs n'auraient plus à faire face à une augmentation des prix quelques mois après les récoltes. Des organisations paysannes de commercialisation et de stockage de céréales avaient vu le jour dans la région de l'Extrême-Nord, mais elles n'ont pas pu se maintenir face au lobby des commerçants à cause du manque de soutien du secteur bancaire et de la faible expérience des organisations paysannes (Mathieu et al., 2003)

\section{La société civile: un acteur émergent}

L'État demeure l'acteur principal pouvant réduire les diverses formes d'insécurité et leurs conséquences. Mais face aux difficultés qu'il rencontre (manque de moyens humains et financiers, perte de confiance entre l'État et les citoyens), on doit aussi s'intéresser à la capacité de la société civile de participer à la résolution des problèmes d’insécurité. Le nord du Cameroun s'est doté, ces 20 dernières années, d'associations de développement local à l'échelle de certains arrondissements et communes, très actives dans le domaine de l'éducation de base et de la santé. Les membres de ces associations relevant de la société civile se sont peu mobilisés sur la question de la violence et des insécurités, probablement faute de savoir-faire. Localement, et à la suite des exactions contre les Mbororo en 2007 et 2008, des associations d'éleveurs ${ }^{4}$ se sont créées d'elles-mêmes afin de contrôler les déplacements des personnes et des troupeaux appartenant à leur communauté.

4 Au Nigéria, par exemple, une organisation regroupant toutes les tribus Mbororo, dénommée Ounguia, lutte contre toutes formes d'exactions. Grâce à cette organisation bien structurée et reconnue par les autorités de ce pays, on enregistre, selon le témoignage des éleveurs, de moins en moins d'agressions, de prises d'otages et de vols de bétail au Nigéria. 
Le travail de fond le plus remarquable dans ce domaine a été réalisé au cours des 20 dernières années par les églises (Comité diocésain de développement, Mouvement Justice et Paix). Ces mouvements ont dénoncé les comportements de certaines autorités traditionnelles et administratives non conformes aux droits de l'homme et ont mis en place un appui juridique aux personnes devant faire face à des injustices. Les bases sociologiques de ces mouvements vont certainement se diversifier à l'avenir. Leur efficacité sera accrue s'ils constituent des réseaux d'associations à différentes échelles d'intervention (village, commune, région) connectés à des réseaux équivalents dans les pays voisins qui éprouvent les mêmes problèmes d'insécurité que le Cameroun. Fournir un appui à ces mouvements et réseaux nécessite des méthodes et des compétences encore trop rares au sein des structures d'appui au développement et à la bonne gouvernance. De plus, ces appuis doivent se faire en totale transparence et en parfaite coordination avec les appuis institutionnels que certains bailleurs de fonds et institutions internationales apportent aux États pour améliorer l'efficacité des services de sécurité et de justice (Jozan et Ray, 2009).

\section{Conclusion}

Insécurité et violence ne sont pas spécifiques au nord du Cameroun (Roitman, 2003 ; Van Dijk, 2009). Le climat d'insécurité semi-permanent au Tchad et surtout en République centrafricaine est à l'origine des déplacements de nombreux éleveurs dans ces pays, d'une envergure plus grande que ceux constatés dans le bassin de la Bénoué au Cameroun (Ankogui-Mpoko, 2009; Seignobos 2009). Les diverses formes d'insécurité observées au nord du Cameroun portent atteinte au bien-être et à la prospérité des ruraux et mettent leurs unités de production dans une situation d'insécurité économique chronique. Pour les éleveurs, la réduction des effectifs de bovins en conséquence des faits de violence entraîne une baisse de revenu. Pour les agriculteurs, la fluctuation des prix des productions et des intrants agricoles, ainsi que le renforcement des aléas pluviométriques, ont accru la tendance à la baisse de leur revenu, déjà amorcée depuis 10 ou 15 ans du fait de la dégradation de la fertilité des sols. Ces ruraux doivent faire face au paiement de taxes et amendes, pas toujours légales, émises par les autorités coutumières et par certains membres des services publics. Cette situation, si elle perdure, pourrait affecter la sécurité alimentaire de l'ensemble de la population de cette région, rurale comme citadine. Par exemple, l'augmentation de la demande en viande et en lait par une population citadine grandissante risque de ne pas être satisfaite et le maintien de la fertilité des sols cultivés risque de ne pas être assuré. Cette paupérisation entraîne certaines personnes dans la délinquance: la communauté Mbororo est concernée, mais les autres communautés n’arrivent pas non plus à juguler ce phénomène.

Cette insécurité plurielle amène, selon les termes de Seignobos et Weber (2002), une «instabilité psychologique» correspondant à l'impossibilité, pour beaucoup de ruraux, de construire un projet d'avenir, en raison de trop d'incertitudes. Il revient alors aux représentants de l'État, mais aussi aux autorités coutumières, d'affirmer leur engagement pour l'éradication des diverses formes d'insécurité. S'il est difficile de trouver des pistes pour résoudre ce climat d'insécurité au nord du Cameroun (fortement influencé par la situation sociopolitique au Tchad et en République centrafricaine), l'analyse de la situation des éleveurs Mbororo montre des signes d'espoir. 
Malgré les difficultés actuelles, ces éleveurs et les agriculteurs valorisent de plus en plus la scolarisation de leurs enfants. Les futures générations seront plus instruites et en mesure de peser contre des positions arbitraires imposées à leurs parents. De plus, même si les problèmes de partage de l'espace et des ressources naturelles sont loin d'être résolus, les insécurités au nord du Cameroun ne sont pas entretenues par l'émergence du communautarisme. Aucune des deux communautés - agriculteurs, éleveurs - n'organise ou ne prêche le pillage de l'autre, comme cela a été observé au nord du Kenya (Galaty, 2002). L'autre signe d'espoir est l'émergence de mouvements issus de la société civile qui œuvrent pour le respect des droits de l'homme et des lois, et pour l'évolution des pratiques de gouvernance. 


\section{Bibliographie}

ABOU ABBA, Abdoulaye, HOFS, Jean Luc et MERGEAI, Guy (2006) Relever les défis environnementaux pour les filières cotonnières d'Afrique de l'Ouest et du Centre. Biotechnologie, Agronomie, Société et Environnement, vol. 10, nº4, p. 351-359.

ANKOGUI-MPOKO, Guy-Florent (2009) Insécurité, mobilité et migration des éleveurs dans les savanes d'Afrique centrale. Dans Savanes africaines en développement: innover pour durer. Actes du colloque de Garoua (Cameroun). Prasac, Ndjamena, Tchad, Cirad, Montpellier (Cédérom).

BANGOURA, Dominique (1996) État et sécurité en Afrique. Politique africaine, $\mathrm{n}^{\circ} 61$.

BEAUVILLAIN, Alain (1989) Nord-Cameroun: crises et peuplements. Rouen, Université de Rouen, thèse de doctorat en lettres et sciences humaines.

BOUTRAIS, Jean (1984) Le Nord du Cameroun: des hommes, une région. Éditions IRD.

COLLIER, Paul et HOEFFLER, Anke (2000) Greed and grievance in civil war. Policy Research, Working Paper no 2355, Washington DC, The World Bank.

COURADE, Georges (2000) Le désarroi camerounais. L'épreuve de l'économie-monde. Paris, Karthala.

DAIROU, Djalla (2007) Rapport sur l'insécurité que vivent les éleveurs dans la province du Nord du Cameroun. MINEPIA. Délégation provinciale de Garoua.

DEVÈZE, Jean-Claude (2006) Le coton, moteur du développement et facteur de stabilité du Cameroun du Nord? Afrique contemporaine, $\mathrm{n}^{\circ} 217$, p. 107-120.

DONGMO, Aimé-Landry (2009) Troupeaux, territoires et biomasses: enjeux de gestion pour un usage durable des ressources au Nord-Cameroun. Paris, AgroParisTech, thèse de doctorat.
DUGUÉ, Patrick, MATHIEU, Bertrand, SIBELET, Nicole, SEUGÉ, C. VALL, Éric, CATHALA, Magali et OLINA, Jean-Paul (2006) Les paysans innovent, que font les agronomes? Le cas des systèmes de culture en zone cotonnière du Cameroun. Dans Jacques Caneil (dir.) Agronomes et innovations. Paris, L'Harmattan, p. 103-122.

DUGUÉ, Patrick, DONGMO, Aimé-Landry et KASPRZYK, Marta (2008) Cameroun: les éleveurs s'organisent. Grain de sel, no 43 , p. 27-28.

DUGUÉ, Patrick, KOSSOUMNA LIBA'A, Natali, DONGMO, Aimé landry, PARESYS, Lise et BALARABE, Oumarou (2011) Territoires d'élevage en zone de savanes au nord du Cameroun: du territoire d'acteurs au territoire d'activité. 13e journée de Géographie tropicale, Université du Mirail, Toulouse.

FOUCAULT, Michel (1992) Le pouvoir, comment s'exerce-t-il? Dans Dominique Colas (dir.) La pensée politique, Paris, Larousse, p. 754-762.

GALATY, John (2002) Vue sur la violence, les frontières du conflit pastoral au Kenya. Anthropologie et Sociétés, vol.26, nº 1 , p. 107-26.

GESCHIERE, Paul (1984) La paysannerie africaine est elle captive? Sur la thèse de Goran Hyden, et pour une réponse plus nuancée. Politique Africaine, no 14 , p. 13-33.

HYDEN, Goran (1983) No shortcuts to progress. African development management in perspective. Berkeley, Los Angeles, University of California Press.

JOZAN, Raphaël et RAY, Olivier (2009) Introduction. De la fragilité à la violence. Les organisations internationales à l'épreuve. Afrique contemporaine, no232, p. 23-38. 
KOSSOUMNA LIBA'A, Natali (2008) De la mobilité à la sédentarisation: gestion des ressources naturelles et des territoires par les éleveurs Mbororo au Nord du Cameroun. Montpellier, Université PaulValéry Montpellier III, thèse de doctorat en géographie.

KOSSOUMNA LIBA'A, Natali, DUGUÉ, Patrick et TORQUEBIAU, Emmanuel (2010) Sédentarisation et renouvellement des pratiques chez les éleveurs Mbororo du Nord Cameroun. Cahiers Agricultures, vol. $19, n^{\circ} 1$, p. 60-67

KOSSOUMNA LIBA'A, Natali et HAVARD, Michel (2006) Mutations de la filière cotonnière dans les provinces septentrionales du Cameroun: perception et stratégies paysannes. Cahiers de géographie du Québec, vol. 50, nº139, p. 65-82.

LE ROY Étienne et TROTHA, Trutz Von (dir.) (1993) La violence et l'État. Formes et évolution d'un monopole. Paris, L'Harmattan.

LE ROY, Étienne (1993) Trente ans de pratiques juridiques à l'ombre de l'État: la domestication du Léviathan. Dans Daniel C. Bach et Anthony Kirk-Greene (dir.) États et sociétés en Afrique francophone. Paris, Economica, p. 41-52.

LEVRAT, Régine (2010) Culture commerciale et développement rural - L'exemple du coton au Nord-Cameroun depuis 1950. Paris, L'Harmattan.

MATHIEU, Bertrand, TEYSSIER, Andrée et ABDOURHAMAME, Nassourou (2003) La sécurité alimentaire: une affaire de paysans. Cahiers Agricultures, vol. 12, n ${ }^{\circ}$, p. 275-281.

M'BIANDOUN Mathurin, GUIBERT, Lorie et OLINA, Jean-Paul (2003) Caractérisation du climat de quatre terroirs de la zone soudano-sahélienne au Nord-Cameroun et conséquences pour l'agriculture. Dans Savanes africaines: des espaces en mutation, des acteurs face à de nouveaux défis. Actes du colloque, mai 2002, Garoua, Cameroun. Prasac, N’Djaména, Tchad Cirad, Montpellier, France (Cédérom).
NAUDIN, Krishna, GOZE, Éric, BALARABE, Oumarou, GILLER, K.E. et SCOPEL, E. (2010) Impact of no tillage and mulching practises on cotton production in North Cameroon: A multi-locational on-farm assessment. Soil \& Tillage Research. p. 68-76.

NDEMBOU, Samuel (1998) Le développement rural dans la plaine de la Bénoué (Cameroun): changements géographiques et permanence sociale. Paris, Université de Paris 1 , thèse de doctorat (remaniée et publiée en 2003).

RAIMOND, Christine, BRETON, Carole, ABOUYA, Alhadji et MOUSSA, Aboubakar (2010) Planification territoriale et accès aux ressources naturelles. Retour sur la démarche participative des opérations de sécurisation foncière dans le Nord Cameroun. Annales de géographie, nº676, p. 639-656.

RIOUX, Jean-François (2001) La sécurité humaine: une nouvelle conception des relations internationales. Paris, L'Harmattan.

ROITMAN, Janet (2003) La garnison-entrepôt: une manière de gouverner dans le bassin du lac Tchad. Critique internationale, $\mathrm{n}^{\circ} 19$ - avril.

ROUPSARD, Marcel (1987) Nord-Cameroun. Ouverture et développement d'une région enclavée. Paris, Université de Paris 10, thèse de doctorat en lettres.

SAÏBOU, Issa (2006) La prise d'otages aux confins du Cameroun, de la Centrafrique et du Tchad: une nouvelle modalité du banditisme transfrontalier. Revue Polis, vol. 13, nos $1-2$.

SEIGNOBOS, Christian (2010) Une négociation foncière introuvable? L'exemple du MayoRey dans le nord du Cameroun. Annnales de géographie, nº676, p. 657-677.

SEIGNOBOS, Christian (2009) Quel avenir pour les Mbororo? [En ligne.] http://www. infosdelaplanete.org/5167/quel-avenirpour-les-mbororo.html/ 
SEIGNOBOS, Christian et WEBER, Jacques (2002) Éléments d'une stratégie de développement rural pour le Grand Nord du Cameroun, vol.1. Rapport principal, Montpellier, CIRAD.

VAN DIJK, Han (2009) L'impact à long terme de la violence sur l'organisation foncière et la sécurité alimentaire. La région du Guéra (centre du Tchad). Dans Savanes africaines en développement: innover pour durer. Actes du colloque de Garoua (Cameroun) 21-24 avril. Prasac, Ndjamena, Tchad, Cirad, Montpellier (Cédérom). 